\title{
How Does a Dipolar Bose-Einstein Condensate Collapse?
}

\author{
John L. Bohn, 1 , Ryan M. Wilson, and Shai Ronen \\ JILA and Department of Physics, University of Colorado, Boulder, \\ CO 80309 USA
}

\begin{abstract}
We emphasize that the macroscopic collapse of a dipolar Bose-Einstein condensate in a pancake-shaped trap occurs through local density fluctuations, rather than through a global collapse to the trap center. This hypothesis is supported by a recent experiment in a chromium condensate.
\end{abstract}

Based on an invited talk in Seminar 6, Physics of Cold Trapped Atoms, at the 17th International Laser Physics Workshop, Trondheim, Norway, June 30- July 4, 2008.

PACS 03.75.Hh, 03.75.Kk

${ }^{1}$ email: bohn@murphy.colorado.edu 
It is not our purpose in this invited presentation to convince the reader of the intrinsic interest of a quantum degenerate gas of dipolar particles. This task has been admirably accomplished in several recent reviews [1, 2, 3, and continues to play out in laboratories and calculations the world over. Suffice it to say that these developments are likely to have an impact on chemistry, condensed matter physics, precision measurements, and quantum information science. Instead, we are interested here in a very practical and immediate concern, namely, the stability of such a gas, and the processes that undermine this stability.

We will focus here on a Bose-Einstein condensate (BEC) of dipolar entities, and assume that the dipoles are strictly aligned in a common direction, which we will identify as the $z$ axis. In this circumstance the interaction between dipoles takes the form

$$
V(\vec{r})=\frac{d^{2}\left(1-3 \cos ^{2} \theta\right)}{r^{3}}+\frac{4 \pi \hbar^{2} a}{m} \delta(\vec{r})
$$

where $d$ is the dipole moment, $r$ the distance between dipoles, and $\theta$ the angle that the intermolecular axis makes with respect to $z$. We have included here also an explicit, isotropic contact interaction that depends on the $s$-wave scattering length $a$. The two-body dipolar interaction is attractive for head-to-tail orientations of the dipoles $\left(\cos ^{2} \theta<1 / 3\right)$, and repulsive otherwise. By contrast, the contact interaction is equally attractive (if $a<0$ ) or repulsive (if $a>0$ ) in all directions.

In BEC of non-polar entities, the stability of the condensate relies on the value of $a$. When $a>0$, the resulting mean-field due to interactions accounts for a kind of outward pressure that inflates the condensate over its non-interacting counterpart; such a condensate is stable. When $a<$ 0 , the mean-field energy generates instead an attractive pressure, which encourages the macroscopic collapse of the condensate into a high-density lump in the center. In this high-density environment, the condensate is prone to loss and heating due to three-body recombination [4. The meanfield attraction is mitigated somewhat by the zero-point energy of the trap in which the atoms are held, however. The result is that in realistic experiments, the BEC can hold a certain critical number of atoms before becoming unstable [4, 5]. Alternatively, for a fixed atom number, the condensate can be made to collapse by tuning $a$ to sufficiently negative values [6].

This consideration of repulsive-versus-attractive interactions raises an interesting issue for the dipolar BEC, since the dipole-dipole interaction is sometimes attractive, sometimes repulsive. In free space without a trapping potential, the attraction is always enough to de-stabilize the gas. However, in a trap, the contribution of the interaction that is dominant depends on the distribution of the dipoles. To see this, consider the schematic in Figure 1. In this figure the solid oval suggests the shape of the trap confining the dipoles, while the shaded region represents the resulting shape of the BEC itself. The trap is harmonic, with an anisotropy defined by the aspect ratio $\lambda=\omega_{z} / \omega_{\rho}$ of its azimuthal to radial trap frequencies.

Consider Fig. 1a), where the trap is nearly spherical, $\lambda \approx 1$. Because the dipole-dipole interaction is attractive for a head-to-tail orientation, 
Figure 1: Schematic view of the collapse of dipolar BEC. In all cases the solid oval represents the trap anisotropy, while the shaded gray area represents the anisotropic condensate density. The arrows merely suggest the direction of dipole polarization. In a), a nearly spherical trap is unstable against distortion of the BEC into a prolate shape,followed by macroscopic collapse. In b), squeezing the trap into a more oblate shape forces the BEC to do the same, allowing a greater number of dipoles before collapse occurs. In c), a very oblate trap becomes unstable against local density fluctuations, in each of which the condensate is effectively prolate as in a)

a)

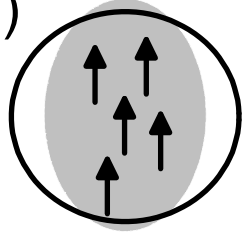

b)

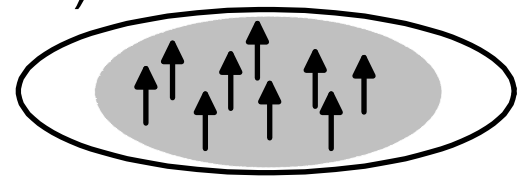

c)

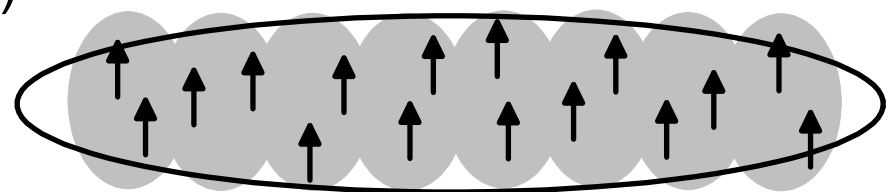


the gas can lower its energy by distorting into a more prolate shape [7, 8]. As the dipole strength grows, this shape becomes more pronounced, emphasizing the attractive part of the interaction, until at last it overwhelms the condensate and collapse occurs. In a spherical trap, and in the absence of a contact interaction, the BEC can stretch to about 1.6 times higher than it is wide, before collapsing.

An obvious way to overcome this instability is to introduce an extra energy cost for the cloud to distort into a prolate shape. To do this, one might contemplate holding the BEC in an oblate trap with $\lambda \gg 1$, as suggested in Fig. 1b). In this case, it might be expected that the gas cannot achieve a prolate shape, regardless of interaction, because it would have to overcome the immense harmonic confinement potential in the $z$ direction. Indeed, estimates based on a gaussian ansatz wave function for the condensate predict that, for $a=0$, the condensate is completely stable beyond a certain aspect ratio, regardless of the number of dipoles in the BEC [8].

Nevertheless, more careful calculations using mean-field theory with the nonlocal potential (1) 9] have suggested that the BEC can become unstable for any aspect ratio, when the dipole strength is large enough [10]. The mechanism by which this happens is illustrated schematically in Fig. 1c). In a very prolate trap, the BEC may reduce its total energy by distorting locally into small, dense clumps. In each such clump the attraction can overwhelm the kinetic energy of trap confinement, just as in the simpler case in Fig. 1a).

At dipole strengths just below the threshold for instability, these clumps appear as distortions of the ground state wave function 10, 11, 12. They are ultimately generated by excited state modes that become soft near the instability, and that we have dubbed "roton" modes for reasons described elsewhere 10, 12. If a just-barely-stable BEC is prepared, and the interaction strength is suddenly ramped into the unstable regime, the BEC will exhibit a dynamical collapse that is seeded by this low-lying roton mode. The reader can access movies of just this sort of collapse at http://grizzly.colorado.edu/ bohn/movies/collapse.htm. On this site there are examples of collapse into both radial and angular excitations. Upon collapsing, the gas can rebound to expand in an anisotropic pattern, as has been found experimentally and theoretically [13].

The stability of a dipolar BEC can be probed experimentally, and indeed this has been done by the Pfau group [14]. This experiment produces a BEC of dipolar chromium atoms in traps of varying aspect ratios. While it is difficult to experimentally change the atom number or dipole strength on demand, the experiment can nevertheless change the sign and magnitude of the $s$-wave scattering length by tuning a magnetic field. An intrinsically less-stable BEC requires a more positive scattering length to ward off its collapse. In theoretical simulations we can of course include the scattering length as well.

The stability diagram probed in Ref. 14 is shown in Fig. 2 on a plot of scattering length at which the BEC goes unstable, versus aspect ratio. In this figure the solid line represents the boundary between stable (above the line) and unstable (below the line) BEC, as determined by the softening of the relevant roton mode. Consider first the small-aspect ratio 
Figure 2: Map of the stability of a dipolar BEC against collapse. Solid line: stable-unstable boundary as computed by mean-field theory, determined by the softening of an excited mode to zero excitation energy. Dashed line: prediction of the stable-unstable boundary based on a gaussian ansatz, which cannot account for the local collapse mechanism in Fig. 1c). Data result from the measurement in Ref. [14, supporting the mean-field calculation over the gaussian ansatz.

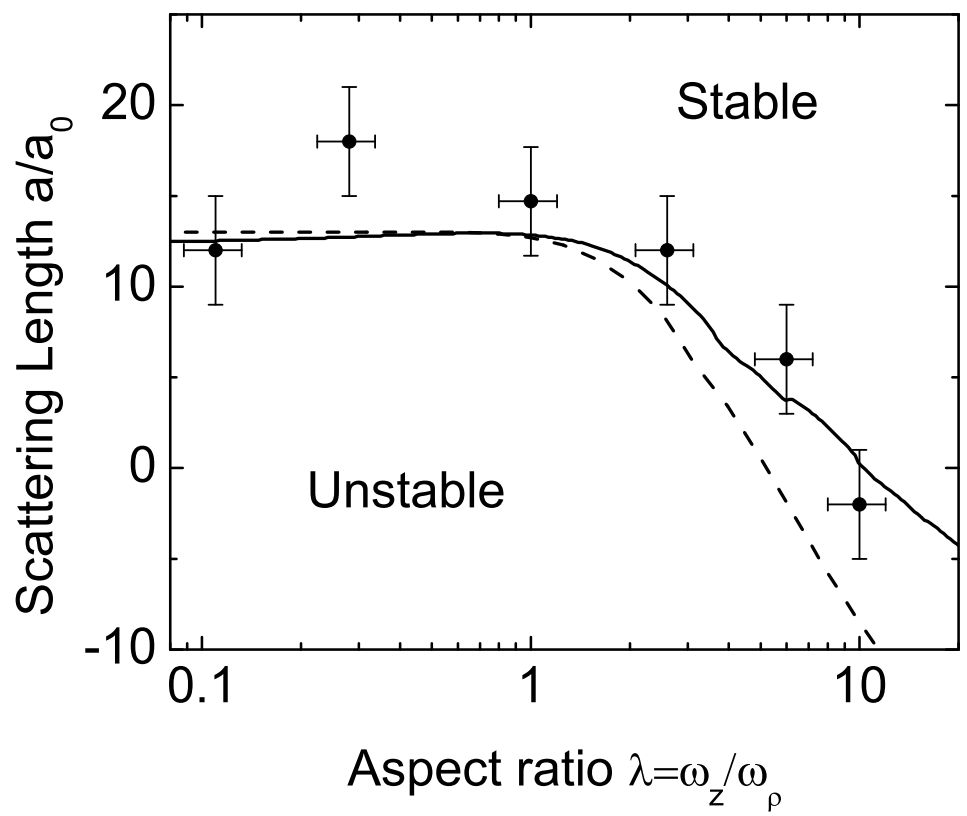

limit (left-hand side of the figure). In this case the trap would readily allow the BEC to distort in a prolate shape as in Fig. 1a); therefore a large, positive scattering length is required to stabilize the gas. By contrast, on the large-aspect ratio (right-hand) side, this distortion is suppressed, and a smaller scattering length is sufficient to stabilize the BEC. Indeed, for the conditions of the experiment, the BEC can sustain a slightly negative scattering length and remain stable. This conclusion would no longer hold, however, for a significantly larger number of chromium atoms [15].

The experimental data are also shown in Fig 2. The agreement with the mean-field theory is quite good, suggesting that this theory remains valid for a chromium gas. In addition, the dashed line shows the prediction for the stability-instability boundary based on a gaussian ansatz wave function. This approximation is accurate for small aspect ratio (prolate traps), but suggests greater stability at high aspect ratio than the meanfield calculation allows. This is because a gaussian wave function cannot 
model local collapse of the kind depicted in Fig. 1c). The data therefore suggest that the mean-field description is the correct one, thus supporting the idea of a collapse due to local density fluctuations.

This work was supported by the U.S. Department of Energy and the National Science Foundation.

\section{References}

[1] G. Kurizki, I. E. Mazets, D. H. O'Dell, and W. P. Schleich, Int. J. Mod. Phys. B 18, 961 (2004).

[2] M. A. Baranov, Phys. Rep. 464, 71 (2008).

[3] T. Lahaye, J. Metz, T. Koch, B. Frölich, A. Griesmaier, and T. Pfau, arXiv:0808.3876 (2008).

[4] R. J. Dodd, M. Edwards, C. J. Williams, C. W. Clark, M. J. Holland, P. A. Ruprecht, and K. Burnett, Phys. Rev. A 54, 661 (1996).

[5] C. C. Bradley, C. A. Sackett, and R. G. Hulet, Phys. Rev. Lett. 78, 985 (1997).

[6] S. L. Cornish, N. R. Claussen, J. L. Roberts, E. A. Cornell, and C. E. Wieman, Phys. Rev. Lett. 85, 1795 (2000).

[7] S. Yi and L. You, Phys. Rev. A 61, 041604(R) (2000).

[8] L. Santos, G. V. Shlyapnikov, P. Zoller, and M. Lewenstein, Phys. Rev. Lett. 85, 1791 (2000).

[9] S. Ronen, D. C. E. Bortolotti, and J. L. Bohn, Phys. Rev. A 74, 013623 (2006).

[10] S. Ronen, D. C. E. Bortolotti, and J. L. Bohn, Phys. Rev. Lett. 98, 030406 (2007).

[11] O. Dutta and P. Meystre, Phys. Rev. A 75, 053604 (2007).

[12] R. M. Wilson, S. Ronen, and J. L. Bohn, Phys. Rev. Lett. 100, 245302 (2008).

[13] T. Lahaye, J. Metz, B. Frölich, T. Koch, M. Meister, A. Griesmaier, T. Pfau, H. Saito, Y. Kawaguchi, and M. Ueda, Phys. Rev. Lett. 101, 080401 (2008).

[14] T. Koch, T. Lahaye, J. Metz, B. Frölich, A. Griesmaier, and T. Pfau, Nat. Phys. 4, 218 (2008).

[15] R. M. Wilson, unpublished. 